\title{
Parallelism in open learning and working environments
}

\section{Rik Min}

Dr Rik Min is in the Faculty of Educational Science and Technology at the University of Twente, PO Box 217, 7500 AE Enschede The Netherlands

\begin{abstract}
One of the main shortcomings of multimedia in open learning environments is the use of monitors. The monitor as currently supplied with the personal computer originates from the world of television and video. Television equipment is extremely suitable for the transmission of one-way messages and also for the presentation of film, video and speakers ('talking heads'). It is also suitable for giving instruction and showing other educational programmes. But the monitor is built as a one-way message medium.

Pictures and images are presented but disappear as soon as other pictures are presented. Thus, a certain measure of short and long memory is required as well as the interpretation of the data and so it remains a linear medium. The organisation of the monitor display is a constant worry in open learning and working environments, because in practice it is continually changing.

This article analyses these shortcomings and describes some solutions according to the concepts and theories of Min, Koopal, Gritter, Struyker Boudier, Coleman, Miltenburg and Van Schaick Zillesen, developed over the last decade (Min, 1992).
\end{abstract}

\section{Working environments}

Interactive working environments such as instruction programs, learning programs and self-organising learning environments require different techniques and methods of presentation on the monitor than linear programs. The problem is not very apparent because most instruction environments resemble linear programs. Selforganising environments such as spread sheets, text processing and programming environments create many minor problems and users employ different tricks to overcome them. For example, many people use old listings and notes on paper beside the computer to help with their navigation when text-processing. Despite the efforts that have been made to dispense with paper in learning environments, we should not believe that everything can be done with electronic materials.

This phenomenon also applies to note-taking, which most users find essential for 
organising their learning processes. Current technology finds it difficult to provide an electronic note-taking facility in parallel with the processes taking place on the monitor.

\section{Learning environments}

In fact, as shown by the discussions on 'learner models' we know very little about learning processes in complicated computerised and non-computerised self-organising environments. Certainly, there are many different processes used by different individuals. A recent research paper of a French group mentioned 250 types of learners in the population of secondary schools.

There are many instructional theories but few about the practice of learning linked to the structure of someone's character. The number of different learner types is undoubtedly influenced by the degree of freedom present in learning. The main characteristic of a classical learning environment is that the pupil can be surrounded by a large amount of informative materials (papers, books, dictionaries, pictures, notebooks, maps, schemes, 'talking heads', etc.).

CAI and CBT are characterised by classical 'programmed instruction'. Information appears on the screen and replaces the previous information which disappears. The user either needs a good memory or has to take notes. However, taking notes is seen by designers of CAI and CBT as a failure in their programs instead of a strength: ignoring the need to take notes and the need to consult other media might be the reason that it has so often failed in this respect. The arrival of multimedia does not solve the problem because the user tries to use more than the electronic media has to offer. There have been many attempts to solve these shortcomings of the video monitor over the last ten years. It seems that human beings retrieve parallel information and like working with lots of things in view.

\section{Parallelism}

Linear media and boring lessons from a teacher are one-way situations while learning environments have a two-way potential. The problem is how to make this clear. Good learning environments require parallel instruction and we can see many such situations all around us in everyday life. Although we are not aware of it, parallelism is a phenomenon that occurs frequently in class and in ordinary life, eg in museums or libraries where one is flooded with information. For example in a classroom the geography teacher will talk about a country while the pupil has a book in front of him; a map will be visible in front of the class; and in a glass case he may even see a display of various pieces of equipment from that country. Although some pedagogues would say that this is not an optimal learning environment, children think differently. They need to learn because they are inspired to do so by other situations. Learning tools such as computer simulation are not meant to be instructive but they should motivate pupils to increase their learning levels and to understand why other earlier (and possibly boring) lessons were required. 
A second example is a museum with a guide or walkman supplied at the entrance. Although this looks linear, it is certainly a form of parallel instruction. Thirdly, there is training in working environments such as learning Word Perfect with headphones and a cassette recorder with instructions. A newspaper is an example of an environment in which people like to have everything in view before they decide what they are going to read. The 'desktop philosophy' of Apple Inc. and Steve Jobs with the WIMPS operating systems and the resulting market for DTP environments has been a tremendous step forward in shaping the monitor's linear character into equipment suitable for more normal two-way human activity. The enormous success of SUN workstations is largely due to the screen diameter which is wider than usual and the monitor's very high activities. Users keep many windows permanently open and on 'stand by' to support their need for a self-structured working environment.

All these examples indicate that efforts are continually made both consciously and unconsciously to satisfy this human need.

\section{The parallel instruction theory}

Instruction Theory for Simulation, the Pi-theory, supposes that for simulation environments the need for instruction is great but parallelism is essential (Min, 1992). Open learning environments for simulation fail without instructions-or with poorly shaped instructions. Many experiments with over one thousand medical students using paper-based instruction materials have been carried out at Limburg University in cooperation with Struyker Boudier (1982). These simulation environments also used two parallel monitors and paper-based instruction materials. Miltenburg with his training course about the dynamics of economics (1988) and Coleman with his simulation programs in university courses for medical students also showed that paper materials are absolutely essential to success (Min, 1993).

The arrival of modern windowing computers such as Macintosh, SUN and Next computers, were a tremendous step forward. Together with Van Schaick Zillsen (1991), the author developed a system for designing simulation programs with many parallel windows, based on Apple's desktop philosophy. A conceptual scheme or map of the mathematical physiological models was used in conjunction with the simulation output. In spite of all this the researchers found to their amazement that paper instruction materials remained a decisive factor.

A cheap and practical method for teachers to produce adapted instruction for simulation programs proved difficult to provide by electronic means. The arrival of a cheap and widely-used instructional authoring system HyperCard, led to a series of experiments with electronic instruction-which proved rather unsuccessful. The researchers concentrated too much on HyperCard's ability to move from one program to the next, and to go from one frame to another, and it became clear, after their experiments, that their learning environment had, unfortunately, become a linear programme. 
After this series of experiments the multi-tasking operating system appeared and Multifinder and System 7 came on the market. The use of multi-tasking proved immediately useful in a working environment but was slower to be adopted in learning environments.

In 1992 the author accidentally found that the multi-tasking operating system was extremely useful for educational computer simulation programs in two respects. Firstly the system allows the simulation part to be made with simulation tools and the instructional part to be made with cheap authoring tools. This situation is very natural for the design of this type of software and is of interest for the 'second' designer - the teacher in school. Both parts are designed by specialists but in entirely different ways and with different tools. Secondly it allows a certain measure of asynchronic working, as with paper instruction materials, because the two parts have stand-alone characteristics. The experiments with Van Schaick Zillsen are now called the 'linear instruction method' (Min, 1992). This is characterised by an introduction to the learning matter so that the pupil can try to master the simulation environment. The instruction program disappears from the screen to be replaced by the simulation, and then the student continues in the instructional program and answers a few test questions on what has been learned. This method is termed the 'sandwich construction'.

\section{What is the aim of this concept?}

The Pi-theory tries to explain why certain learning environments do not result in good learning behaviour whereas others do. It is a theory about shaping instruction with simulation and it possibly explains why MS-DOS computers are not really suited to the creation good simulation environments with electronic instructions. The theory explains a number of things including:

- Why simulations with paper instruction materials are so popular?

- Why do open simulation environments without good parallel instruction not work?

- Why are some students unable to work in their rooms surrounded by papers, notebooks etc.?

- Why have the big screens of SUN workstations, with many windows proved to be such an excellent working environment for many students?

- Why do designers always put so much information into one frame on a screen? (This is proof that in principle parallel presentation of information or instruction is essential);

- Why in this computer and television era, is an illustrated textbook still a satisfactory learning tool? Are books irreplaceable after all?

- Why does courseware on MS-DOS computers frequently not work for a large proportion of students?

\section{Conclusions}

Frequently, through the years of research and development projects in the field of simulation, it is has been shown that the media were lacking in the classical skills of 
Iaboratory presentation. Computer simulations have generally proved more successful when accompanied by paper notebooks. Computer based materials that did not take parallel instruction theory into account, failed time and again until the arrival of the multi-tasking operating systems. Total electronic learning needs a user environment in which things can be kept in view for an unlimited period of time. Most people do not need the interaction between one object and the next. They like asynchronic objects.

People are best motivated to receive instruction when they can decide for themselves at what time and how much they need it. With linear instruction, the instruction always arrives at the wrong time and in the wrong quantity. It has disappeared by the time it is needed most. Most interactive media prove in practice to be a collection of often linear concepts, with consequent drawbacks. Our first experiment with sequential, electronic instructions (the 'sandwich construction') gave us the clue.

In the field of instrumentation technology, creating a simulation learning environment with a specific tool equipped for the provision of simulations, and the instruction environment with a second specific instruction tool is more practical than using one authoring system for both simulations and instruction.

The MS-DOS/Intel concept is too oriented to informaticians and is not suited to the learning attitudes of children and adults. MS-DOS and even Windows 3.1 are technically incomplete for the creation of quick multi-windowing, multi-tasking environments based on the Pi-theory and lag behind Macintosh and Next computers. The desktop video system and the natural way in which they handle windows on the Motorola/ 68000 computers is inimitable. IBM's most recent manoeuvres to purchase the Next software concept of Steve Jobs looks promising for the not too distant future.

\section{References}

Ambron S and Hooper K (eds) (1990) Learning with Interactive Multimedia; Developing and Using Multimedia Tools in Education. Microsoft Press, Redmond, Washington. ISBN 1-55615-282-5. Gritter H (1993) Designing, realisation and evaluation of ISAV (Instructional Support to ARCVIEW); a CBT program for the program ARCVIEW MSc thesis (in Dutch) University of Twente (in cooperation with the University of Southampton) Enschede.

Koopal W (1993) Design, realisation and evaluation of learning environments for simulation MSc thesis (in Dutch) University of Twente, Enschede.

Min F B M (1992) Parallel Instruction, A Theory for Educational Computer Simulation Interactive Learning International 63 , pp 177-183.

Min F B M (1993) Computer Simulation as an Educational Tool (Chapter 2). The heart as pump. The computer simulation program CARDIO (Chapter 10). Fluid volumes: the computer simulation program FLUIDS (Chapter 19) in Möller D and van Wijk van Brievingh $\mathrm{R}$ (eds) Modelling and simulation in physiology and biomedical engineering on a Personal Computer Springer Verlag Berlin; (Editors:) ISBN 3-387-97650-7/ISBN 3-540-97650-7.

Min F B M and Struyker Boudier H A J (1982) Computer simulation programs in problem oriented medical learning at the university of Limburg Computers and Education 6 153-158. Schaick Z P van (1990) Methods and techniques for the design of educational computer simulation programs and their validation by means of emperical research $\mathrm{PhD}$ thesis, University of Twente. ISBN 90-9003874-4. 